\title{
Delta and Notch promote correct localization of IrreC-rst
}

\begin{abstract}
Dear Editor,
The Notch signal transduction pathway has been implicated in a variety of cell fate decisions throughout development. In the developing Drosophila retina, the Notch receptor and its ligand Delta are required to organize the interommatidial lattice through selective programmed cell death (PCD). ${ }^{1,2}$ Another transmembrane molecule required for $P C D$ in the Drosophila retina is the immunoglobulin superfamily member Irregular chiasmC-roughest (IrreC-rst). ${ }^{3-5}$ Loss-of-function mutations in irreC-rst lead to a block in PCD during retinal development, resulting in a rough eye phenotype in the adult. In addition, the irreC-rst ${ }^{C T}$ mutation, which results in truncation of the IrreC-rst intracellular domain, is associated with reduced IrreC-rst protein at the plasma membrane and increased IrreC-rst within intracellular vesicles. ${ }^{4,5}$ Retinal PCD is lost, indicating that proper subcellular localization of IrreC-rst may be an important factor in permitting or promoting the death process. The mechanisms by which Notch signaling and IrreC-rst regulate PCD are unclear.
\end{abstract}

In a recent genetic screen (Tanenbaum et al, submitted) and in direct tests, Delta $(D I)$ mutations were identified as dominant enhancers of the rough eye phenotype conferred by the hypomorphic allele irreC-rst ${ }^{3}(n=7$ DI mutant lines; Figure $1 \mathrm{~A}-\mathrm{C}$ ). These observations demonstrate that Delta and irreC-rst interact genetically to execute PCD, and motivated us to examine further the relationship between Delta/Notch signaling and IrreC-rst.

In a wild-type retina, differentiation of the primary pigment cells $\left(1^{\circ} \mathrm{s}\right)$ in the young pupa is followed by selective removal of approximately one-third of the neighboring interommatidial precursor cells by $\mathrm{PCD} .^{3,6}$ The remaining interommatidial precursor cells are organized concurrently into a hexagonal array and differentiate as optically-insulating secondary/tertiary pigment cells $\left(2^{\circ} / 3^{\circ} \mathrm{s}\right.$; Figure $\left.1 \mathrm{D}\right)$. During the state of $\mathrm{PCD}$, IrreC-rst protein is expressed at high levels in the $2^{\circ} / 3^{\circ} \mathrm{S}$ and at low levels in the $1^{\circ} \mathrm{s}$. It is localized subcellularly along the border between the $2^{\circ} / 3^{\circ} \mathrm{S}$ and the $1^{\circ} \mathrm{s}$, and between the two $1^{\circ} \mathrm{s}$ of each ommatidium ${ }^{5}$ (Figure 1D-F). This subcellular localization of IrreC-rst is lost in two Notch alleles $\left(N^{f a-g 2}, N^{f a-s w b}\right)$ that reduce Notch activity specifically in the young pupal retina during pigment cell differentiation; however, these alleles also result in a loss of $1^{\circ} \mathrm{s}$ and a block in PCD. ${ }^{1,5}$ Mislocalization of IrreC-rst could represent an indirect effect due to defects in $1^{\circ}$ differentiation or PCD. Alternatively, Notch protein or signaling could affect more directly the localization of IrreC-rst.

To distinguish between these possibilities, we utilized temperature-sensitive alleles to reduce Delta and Notch function specifically during the stage of PCD, subsequent to formation of the $1^{\circ} s^{1,2,7}$ Flies heterozygous for the temperature sensitive allelic combination $D I^{R F} / D I^{6 B}$ were shifted to the non-permissive temperature. A temperature shift of $6 \mathrm{~h}$ duration resulted in a loss of IrreC-rst localization: the IrreC-rst protein was redistributed throughout the apical surfaces, revealing the boundaries between $2^{\circ} \mathrm{S}$ and $3^{\circ} \mathrm{S}$ (Figure $1 \mathrm{G}$ ). Similarly, shifting $N^{t s 1}$ pupae to the non-permissive temperature for $8 \mathrm{~h}$ resulted in a redistribution of IrreC-rst protein (Figure $1 \mathrm{H}$ ). In parallel control experiments, redistribution of IrreC-rst protein was not observed in temperature shift experiments with wildtype or with either $D I^{R F}$ or $D I^{6 \mathrm{~B}}$ in trans to a normal copy of Delta (Figure 1E,F), in $D I^{R F} / D I^{6 B}$ flies shifted for intervals ranging from 0 to $4 \mathrm{~h}$, or in unshifted $N^{t s 1}$ flies (data not shown).

The observed redistribution of the IrreC-rst protein was not an indirect effect of a loss of PCD in the $D I^{R F} / D I^{6 B}$ and $N^{t s 1}$ backgrounds: when PCD was blocked by directed expression of the activated dRas1 isoform dRas $1^{\text {val12 } 8,9}$ or the baculovirus caspase inhibitor p35, ${ }^{10}$ IrreC-rst localization was unaffected (Figure 11,J). This indicates that the redistribution of IrreC-rst protein is a specific effect of the reduction of Notch or Delta activity or protein and not a result of incorrect $2^{\circ} / 3^{\circ}$ patterning. Correct localization of IrreC-rst is likely important for its normal function, ${ }^{5}$ and it will be interesting to determine the mechanism by which it is regulated through Delta and Notch. 
1012
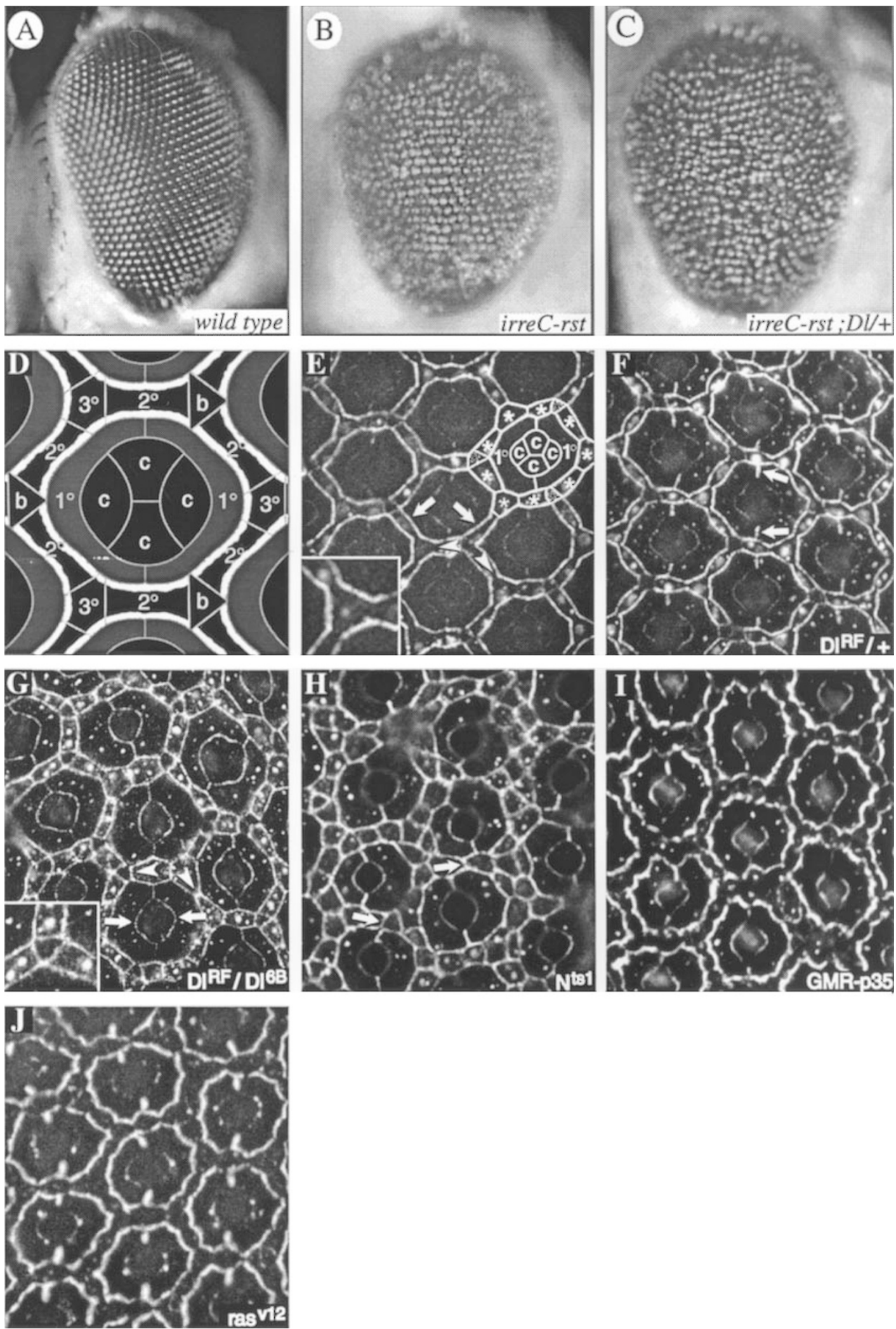

Figure 1 The rough eye phenotype of irreC-rst ${ }^{3}$ is enhanced by Delta ${ }^{E 58}$. (A) A wild-type adult eye contains approximately 750 ommatidia arranged in straight lines along multiple axes. (B) A y irreC-rst ${ }^{3} ; d p^{o v} ; p^{p}$ adult eye has a mildly altered pattern of ommatidia compared to wild-type; careful inspection reveals an occasional 'jog' in the ommatidial rows. (C) These alterations are enhanced significantly in an irreC-rst ${ }^{3}$; Delta ${ }^{E 58} /+$ adult eye. Six other $D /$ alleles were identified as dominant enhancers of irreC-rst ${ }^{3}: D I^{J 17}, D I^{J 111}, D I^{K 75}$ (Tanenbaum et al, submitted), $D I^{H D 82}, D I^{S 130403}$ and $D I^{S 148504}$. In addition, $D I^{E 58}, D I^{J 17}, D I^{K 75}, D I^{J 111}$ and $D I^{H D 82}$ were observed to enhance irreC-rst ${ }^{\mathrm{UB883}}$ and irreC-rst ${ }^{1 / 34}$; the other alleles were not tested. (D-J) Localization of IrreC-rst protein in pupal retinae. In (E-H and $\left.\mathbf{J}\right)$ 
flies received a heat shock (see below), were dissected immediately, and immunostained using the IrreC-rst-specific antibody mAb $24 A 5.1 .^{11}$ (D) Schematic of the apical surface of a wild-type pupal retina. One central ommatidium is shown, and is labeled to indicate the non-neuronal cone cells (c), the optically-insulating primary pigment cells $\left(1^{\circ}\right)$, the optically-insulating secondary $\left(2^{\circ}\right)$ and tertiary $\left(3^{\circ}\right)$ pigment cells, and bristles (b). The photoreceptor core consisting of eight neurons is not visible in this view. Subcellular localization of IrreC-rst protein in $2^{\circ} / 3^{\circ} \mathrm{s}$ is indicated by white shading. For clarity, IrreC-rst expression in $1^{\circ} \mathrm{s}$ is omitted. (E) Wild-type control retina from an animal aged until $44 \mathrm{~h}$ after puparium formation (APF; $18^{\circ} \mathrm{C}$ ), shifted to $32^{\circ} \mathrm{C}$ for $6 \mathrm{~h}$ and dissected immediately. One ommatidium and its associated $2^{\circ} / 3^{\circ}$ lattice is traced (compare with (D)); the cone cells (c), $1^{\circ} \mathrm{s}, 2^{\circ} / 3^{\circ} \mathrm{s}$ (white asterisks), and bristles (filled asterisks) are labeled. Note the distinct localization of IrreC-rst protein between $1^{\circ} \mathrm{s}$ and $2^{\circ} / 3^{\circ} \mathrm{s}$ (e.g., arrows) but not, for example, between two $2^{\circ} / 3^{\circ} \mathrm{s}$ cells (arrowheads). An enlarged view of a single centra $2^{\circ} / 3^{\circ} \mathrm{s}$ and three $2^{\circ} / 3^{\circ} \mathrm{s}$ radiating from it are shown in the inset; the boundaries between $2^{\circ} / 3^{\circ} \mathrm{s}$ cannot be seen due to a lack of IrreC-rst protein (compare with (G)) The vesicles within the $2^{\circ} / 3^{\circ} \mathrm{s}$ are likely multivesicular bodies, sites of protein internalization and turnover. ${ }^{12}(\mathrm{~F}) \mathrm{DI}{ }^{R F} /+\mathrm{control}$ retina. A $6 \mathrm{~h}$ heat pulse, performed as in $(\mathbf{E})$, had no effect on IrreC-rst localization. Subcellular localization between two $1^{\circ} \mathrm{s}$ is more obvious than in (E) (arrows). (G) $D I^{R F} / D I^{6 B}$ retina following a $6 \mathrm{~h}$ heat pulse as in (E). IrreC-rst protein is no longer localized primarily at the border between $1^{\circ} \mathrm{s}$ and $2^{\circ} / 3^{\circ} \mathrm{s}$ but is distributed equally throughout the $2^{\circ} / 3^{\circ}$ cells. This can be seen most easily with the staining between two $2^{\circ} / 3^{\circ} \mathrm{s}$ (e.g., arrowheads). The inset contains an enlarged view of a central $2^{\circ} / 3^{\circ}$; its boundaries with three neighboring $2 \% 3^{\circ}$ s can now easily be seen due to re-localization of IrreC-rst (compare with (E)). The $1^{\circ}$ membranes that directly abut cone cells (arrows) also appear to have increased levels of IrreC-rst staining at the expense of regions that contact a neighboring $1^{\circ}$ (compare with arrows in $(\mathbf{F})$ ). $(\mathbf{H}) N^{t s} 1$ retinae that received an $8 \mathrm{~h}$ heat pulse demonstrated a similar redistribution of IrreC-rst as $D I^{R F} / D I^{6 B}$ retinae; arrows indicate two examples. The additional $2^{\circ} / 3^{\circ}$ cells - the result of a block in $\mathrm{PCD}-$ are particularly evident in this retina; they can be recognized as $2^{\circ} / 3^{\circ} \mathrm{s}$ based on their small apical profile and their position between ommatidia (see also ${ }^{1}$ ). Notch ${ }^{\text {ts } 1}$ pupae were aged until $42 \mathrm{~h} \mathrm{APF}$ at $18^{\circ} \mathrm{C}$ or $21 \mathrm{~h} \mathrm{APF}$ at $25^{\circ} \mathrm{C}$ prior to the $8 \mathrm{~h}, 32^{\circ} \mathrm{C}$ heat pulse. (I) Expression of the caspase inhibitor p35 throughout the retina leads to a loss of programmed cell death, but had no effect on the localization of IrreC-rst protein. Shown is a retina from $G M R-p 35$ at $28 \mathrm{~h}$ APF ( $\left.25^{\circ} \mathrm{C}\right)$. (J) Retina from $h s$ dRas $1^{\text {val12} / C y O}$ following a $1 \mathrm{~h}$ heat shock $\left(37^{\circ} \mathrm{C}\right)$ at $26 \mathrm{~h} \mathrm{APF}\left(25^{\circ} \mathrm{C}\right)$ and dissected immediately. Localization of IrreC-rst is similar to wild-type

We thank K Fischbach, B Hay, M Muskavitch, the Szeged Stock Center and the Umea Stock Center for supplying fly stocks, and K Fischbach for providing mAb 24A5.1. We also thank $\mathrm{R}$ Kopan and M Marra for comments on the manuscript. This work was supported by grant $\mathrm{NIH}$ R01EY11495; C Baker Brachmann was supported by a fellowship from Damon Runyon.

\section{SM Gorski ${ }^{1}$, C Baker Brachmann ${ }^{1}$, SB Tanenbaum ${ }^{1}$ and $R L$ Cagan $^{\star 1}$}

${ }^{1}$ Department of Molecular Biology and Pharmacology, Washington University School of Medicine, Campus Box 8103; 660 South Euclid Avenue, St. Louis, Missouri 63110, USA

*Corresponding author: E-mail: cagan@molecool.wustl.edu
1. Cagan RL and Ready DF (1989) Genes Dev. 3: 1099-1112

2. Parks AL et al. (1995) Mech. Dev. 50: 201-216

3. Wolff T and Ready DF (1991) Development 113: 825-839

4. Ramos RG et al. (1993) Genes Dev. 7: 2533-2547

5. Reiter $C$ et al. (1996) Development 122: 1931-1940

6. Cagan RL and Ready DF (1989) Dev. Biol. 136: 346-362

7. Shellenbarger DL and Mohler JD (1978) Dev. Biol. 62: 432-446

8. Fortini ME et al. (1992) Nature 355: 559-561

9. Miller DT and Cagan RL (1998) Development 125: 2327-2335

10. Hay BA et al. (1994) Development 120: 2121-2129

11. Schneider T et al. (1995) Neuron 15: 259-271

12. Cagan RL et al. (1992) Cell 69: 393-399 